\title{
Integration of a Purchase Porter System into a VO Developed by PANGEA
}

\author{
Katsumi Harashima ${ }^{1}$, Hiroki Nakazaki ${ }^{1}$, Pablo Chamoso ${ }^{2}$, \\ Javier Bajo $^{3}$, and Juan M. Corchado ${ }^{2}$ \\ ${ }^{1}$ Osaka Institute of Technology, Osaka, Japan \\ ${ }^{2}$ Computer and Automation Department, University of Salamanca, Spain \\ ${ }^{3}$ Department of Artificial Intelligence, Technical University of Madrid, Spain \\ \{harashima, e1611071\} @oit.ac.jp, \\ \{chamoso, corchado\}@usal.es, jbajo@fi.upm.es
}

\begin{abstract}
Practical applications of multi-agent systems have acquired a growing relevance during last years. This kind of system is based on distributed artificial intelligence and they pay special attention to organizational concepts, This allows a more realistic design of artificial societies. In this paper, we are presenting a multi-agent system based on virtual organizations, which has been specially designed to model a Purchase Porter System aimed at helping clients of shops to transport their shopping. The system establish agreements to transport shopping to the more appropriated exists for the client. The system has been tested in a simulated environment and the initial results are promising.
\end{abstract}

Keywords: purchase porter system, multi-agent systems, virtual organizations.

\section{$1 \quad$ Introduction}

Even though e-commerce is the most popular commercial method over the last few years, most consumers still go shopping at commercial centres.

A large part of the success of these shopping centres is based on the wellbeing offered to clients. According to [1], six are the main factors that affect comfort: functionality, convenience, safety, leisure, atmospherics and self-identification.

However big malls have disadvantages. For example, clients usually report they find difficult taking bags and shopping items from one shop to another. Obtaining a solution for this problem requires designing new mechanisms for the acquisition and management of huge amounts of information about the environment.

Multi agent systems (MAS), used as a way to create artificial societies, have achieved great importance during the last years [2][3].

MAS are one kind of distributed artificial intelligence in which autonomous and individual agents collaborate and cooperate within an artificial society to achieve defined objectives. These MAS incorporate advanced reasoning models as well as learning and adaptation capacities.

One of their many characteristics is the ability to represent interaction models, close to the ones found in human societies [4]. Nonetheless, they do not consider organizational aspects such as rules or regulations. 
Therefore, it is necessary to utilize virtual agent organizations to implement a realistic artificial society. These organizations allow to represent regulatory and management aspects, which is a much better implementation, being a more efficient and practical solution.

In the framework of this paper, it is proposed a help system to aid customers in shopping malls, designed as a MAS based on virtual organizations (VO), in which every virtual organization represents each different area of the commercial centre. At least, one porter agent will exist in each organization and it will be responsible for receiving all customer purchases, as well as delivering them in a convenient place at a scheduled time.

To accomplish the implementation of this system, PANGEA [5], an agent platform, has been chosen as it was developed by the participants of this work. This is important, as it was mentioned before, for resembling the system architecture to the real structure of shopping malls. Although, virtual organizations are self-adapting systems that suit specific situations automatically, without needing human interaction.

Through this article, the problem described in section 2 will be explained with more detail. In chapter 3, the suggested solution, based on virtual organizations, is introduced and then, its results and conclusions in section 4.

\section{Review of the State of the Art}

We refer to MAS when two or more agents are able to collaborate for solving a problem [6]. Concretely, one of them is required to be autonomous, and at least, there must exist a relation between two agents in which one of those satisfies the objectives of the other.

The main characteristic is that every agent focus in its own individual task, although the data they use is decentralized across the system. So, each agent must establish their goals and plans to meet their objectives, always taking into consideration the environment where it is located. However, to accomplish those tasks, agents have to work coordinately, so this is why the global system needs to implement communication and control mechanisms.

In order to implement global system control, modelling its structure as a set of VOs can be very helpful. VO are sets of individuals and institutions that need to coordinate their resources and services within some institutional bounds [7]. Therefore, agentbased virtual organizations hold a social structure and a set of rules which regulate the interaction among the different agents. In addition, they have to own a coordination mechanism to determine the way agents fulfill their tasks.

There are several previous works which focus their case studies in the application of models with agent-based virtual organizations to shopping malls, i.e. the one explained in reference [8]. In that case, a MAS was responsible of managing and planning routes to clients making use of THOMAS architecture [9]. 


\section{A VO-Based Purchase Porter Multi-agent System}

In the last few years, many large-scale commercial institutions have found important business opportunities by creating shopping malls with a great number of brand stores and services such as cinemas, restaurants or skating rinks. This is one of the reasons why malls are successful.

When people go shopping at commercial centres, they buy products in different stores, carrying more and more items from one shop to another. As purchases increase, customers feel tired and uncomfortable.

For the purpose of solving the problem mentioned above, we have proposed a purchase porter system with multi-agent technology. In this porter system, individual porter agents have their own workspace. They receive purchases from each store and then, they carry them to the exit to be on time for the desire collection time.

When a porter agent of a certain area receives a transportation request from a shop, products are delivered from the shop location to the agent position. If clients desire to pick the items at the exit of this area, the porter agent transport them to the specified place on time.

Otherwise, the agent communicates with the representatives of adjacent areas. Internally, the best possible route is calculated considering the destination, time remaining and the priority of other requests.

This way, products are optimally carried across several areas by delegating the transportation task among several porter agents, each one responsible of its own workspace.

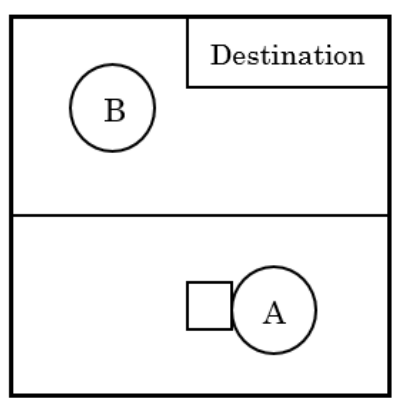

(a)

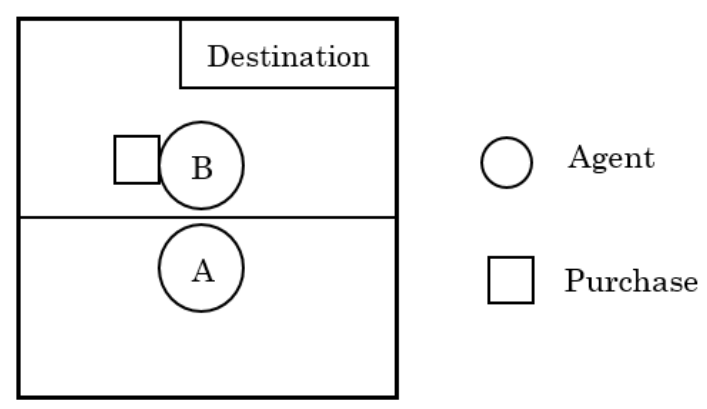

(b)

Fig. 1. Transfer of purchase

Figure 1 shows the transfer of purchases among agents. In Fig 1(a), the initial situation is represented, having the agent $\mathrm{A}$, a purchase needed to be delivered at the destination point. As this location is at the exit of agent B workspace, A gives the purchase to agent B, Fig 1(b).

With this system, clients do not need to take items with them every time they enter a new shop. Also, purchases are collected whenever the client wants and in the exit they choose. Usually, customers would like to pick all the items when they return home. 


\subsection{Features of the Purposed Porter System Are as Follows:}

\subsubsection{Environment}

The environment has several stores and exits.

- Store

In each store, transportation of purchases occurs constantly.

- Destination

Every destination is located on the outer circumference of the environment and they become the final destination of the purchase.

\subsubsection{Purchase}

Every purchase has information about a collection point (exit) and time, both set by the customer. Our system adds a priority calculated according to the remaining time and distance to the destination.

Table 1 shows priority for the destination time. Our system attaches a priority so the system is working full time. The shorter the remaining time, the higher the priority is.

Table 2 shows priority for the distance to the destination. It includes a priority system so that the distance becomes as long as possible. When a distance is shorter, the associated priority to the distance is larger, due to efficiency.

Porter agents are able to transport purchases by evaluating these priorities without being late for a time limit.

\subsubsection{Agent}

An individual porter agent has a certain workspace and it can only move in its own area. They receive the purchases of a shopper from each store. Then they carry those purchases to the destination to be ready for collection on time.

Table 1. Priority for remaining time

\begin{tabular}{|c|c|c|c|c|c|c|}
\hline remaining time $t$ & $t>50$ & $50>t$ & $40>t$ & $30>t$ & $20>t$ & $10>t$ \\
\hline$m$ & 1 & 2 & 3 & 4 & 5 & 6 \\
\hline time limit $f(m)$ & 3 & 11 & 26 & 50 & 85 & 133 \\
\hline
\end{tabular}

Table 2. Priority for distance

\begin{tabular}{|c|c|c|c|c|c|c|}
\hline distance $l$ & $l>10$ & $10>1$ & $8>1$ & $6>1$ & $4>1$ & $2>1$ \\
\hline$n$ & 1 & 2 & 3 & 4 & 5 & 6 \\
\hline distance priority $g(n)$ & 2 & 8 & 20 & 40 & 70 & 112 \\
\hline
\end{tabular}


Each agent behaves as described below:

1. First, it receives information about a new purchase (transport request) from a shop in its workspace.

2. It compares the new purchase priority with the others queued.

3. If the new priority is higher, it has to move to the shop which sent the information and receive the items. Otherwise, it does not do anything.

4. If the destination of the purchase with the highest priority is within the agent's workspace, then it takes it there. Otherwise, jump to step 6.

5. If a new purchase in its workspace is received, jump to step 1.

6. If the adjacent agents are located near the destination, it gives the purchases to those agents only if the requests in their queues have lower priorities.

7. If an agent does not have any purchase, it moves to the center of its workspace and then starts again from step 1. If not, it jumps to step 5.

\subsection{Integration in a Multi-agent Platform (PANGEA)}

To implement this system, it has been decided to use multiagent architecture PANGEA as it is based in organizational concepts. This, regarding its similarity with the real world, is going to permit modelling and implementing the large range of different structures shopping malls have. Although, as a rule, they usually share similar organizations.

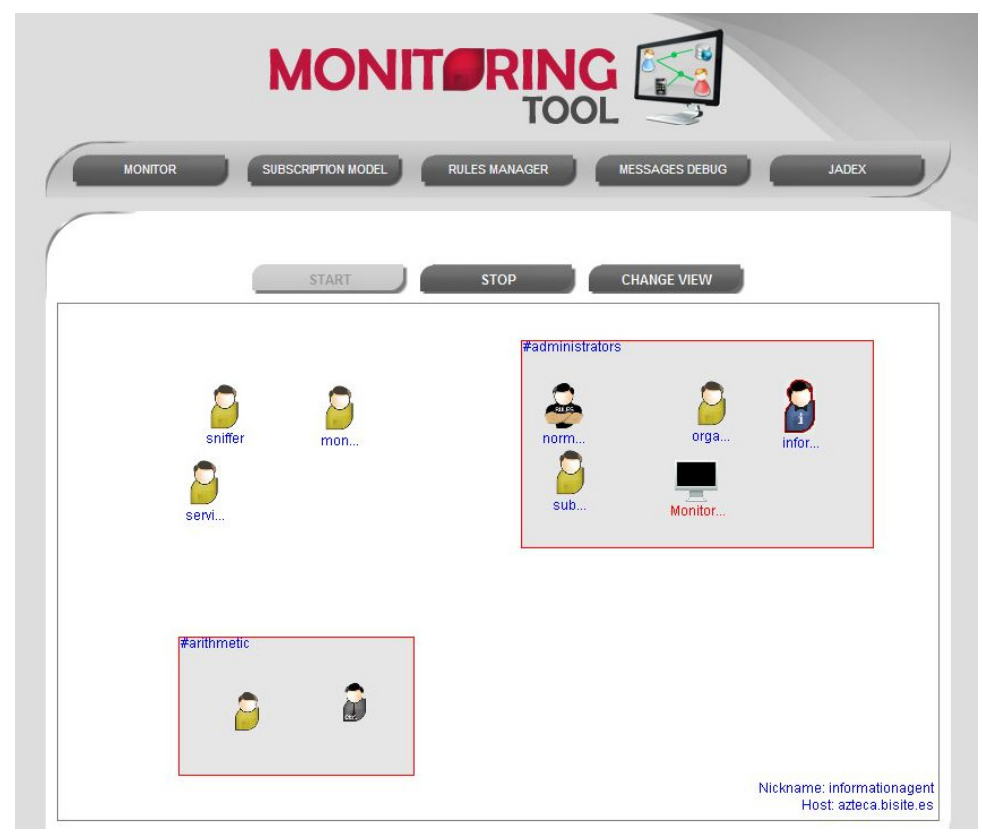

Fig. 2. Image showing the 'Monitoring tool' with the basic agents provided by PANGEA 
This characteristic allows to improve the control of each node in the system in an individual or collective way.

Furthermore, this is fundamental when controlling and monitoring the state of the client purchases in every moment. This process is accomplished by the 'Monitoring Tool' which allows to keep and visualize records of everything that has happened in the system. It also enables to define a set of rules which are fundamental during the process of implementation.

Several specifically designed and structured agents have been added to control agents, which the architecture presents for using basic functions. These new agents develop the case study introduced in this article.

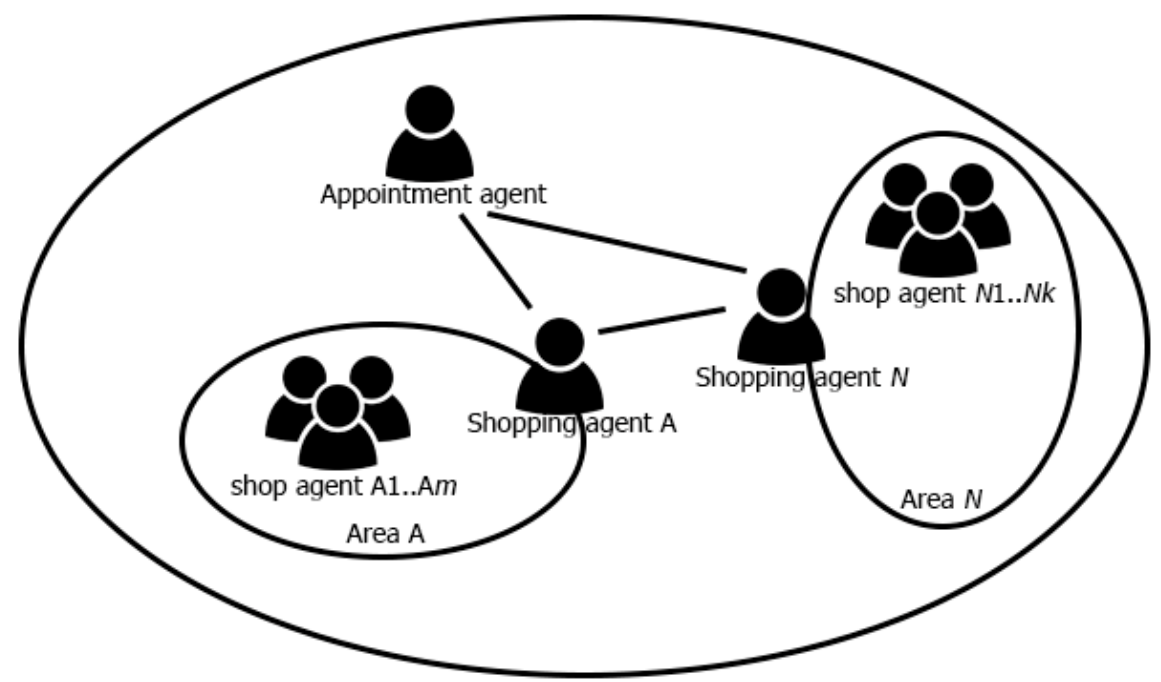

Fig. 3. Scheme showing the subsystem developed

In figure 3, it is shown a scheme representing the subsystem structure for the case study proposed. In it, there are three virtual organizations as an example, but there will be as many as areas defined in the shopping mall plus one. New areas are defined by creating new organizations. This task is performed by the organization agent which is provided by PANGEA.

In each organization associated to an area, there must exist an agent for every shop existing in that area. This agent will notify the shopping agent each time a new purchase is completed in the shop it represents.

Also, every shopping agent is located in a virtual organization together with the appointment agent, which is in charge of managing all system priorities as it has been explained before.

In order to check whether which areas (represented as organizations in PANGEA) are adjacent among them, several XML rules are defined. They indicate which organizations can communicate among them (adjacent organizations) and those which 
cannot. This process is carried out by the PANGEA rule agent, using its monitoring tool following the scheme below:

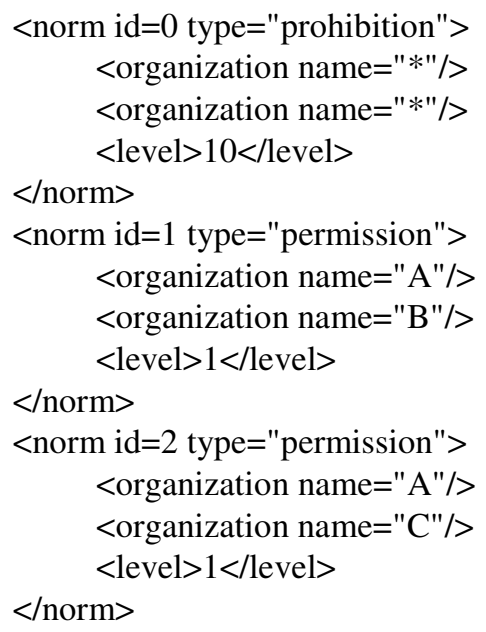

This would mean, as expressed in the rule with identifier equal to 0 , that no organization could communicate with others, unless there would be rules with higher priority (lower level) indicating the opposite. This is the case for rules with id 1 and 2 which express that organizations (areas) A-B and A-C are adjacent.

\section{$4 \quad$ Results and Conclusions}

This article introduces a new porter system with multi-agent systems and virtual organizations. The main focus of this article is to provide a customer-driven solution in large scale commercial institutions. However, satisfying all clients may be a complicated task, due to its high number. Despite this, the system is able to transport almost all purchases to the exit on the specified time. This is possible thanks to the proposed priority queue.

However, in our system, the stores request delivery of purchases at a constant interval. But this is unrealistic. Therefore, our future work is to implement several improvements to address a real flow of purchases over time.

Regarding the use of virtual organizations, the architecture is organized in a way that resembles a real shopping mall. This way, areas and agents are distributed easily. Likewise, PANGEA features fit perfectly to create organization-based structures, permitting control and monitoring all moves occurred. This architecture presents an adaptive and scalable design capable of adjusting itself to new conditions, being applicable to any commercial setting. 


\section{References}

[1] El Hedhli, K., Chebat, J.C., Sirgy, M.J.: Shopping well-being at the mall: Construct, antecedents, and consequences. Journal of Business Research 66(7), 856-863 (2013)

[2] Lane, J.E.: Method, Theory, and Multi-Agent Artificial Intelligence: Creating computer models of complex social interaction. Journal for the Cognitive Science of Religion 1(2), 161-180 (2014)

[3] Fan, Z., Duan, W., Chen, B., Ge, Y., Qiu, X.: Study on the method of multi-agent generation algorithm within special artificial society scene. In: 2012 UKACC International Conference on Control (CONTROL), pp. 1076-1081. IEEE (September 2012)

[4] Schreinemachers, P., Berger, T.: An agent-based simulation model of human-environment interactions in agricultural systems. Environmental Modelling \& Software 26(7), 845-859 (2011)

[5] Zato, C., Villarrubia, G., Sánchez, A., Bajo, J., Corchado, J.M.: PANGEA: A New Platform for Developing Virtual Organizations of Agents. International Journal of Artificial Intelligence $^{\mathrm{TM}} 11(\mathrm{~A} 13), 93-102$ (2013)

[6] Mas, A.: Agentes software y sistemas multiagente: conceptos, arquitecturas y aplicaciones. Prentice Hall (2005)

[7] Boella, G., Hulstijn, J., Van Der Torre, L.: Virtual organizations as normative multiagent systems. In: Proceedings of the 38th Annual Hawaii International Conference on System Sciences, HICSS 2005, pp. 192c-192c. IEEE (January 2005)

[8] Rodriguez, S., Julián, V., Bajo, J., Carrascosa, C., Botti, V., Corchado, J.M.: Agent-based virtual organization architecture. Engineering Applications of Artificial Intelligence 24(5), 895-910 (2011)

[9] Julian, V., Rebollo, M., Argente, E., Botti, V., Carrascosa, C., Giret, A.: Using THOMAS for Service Oriented Open MAS. In: Kowalczyk, R., Vo, Q.B., Maamar, Z., Huhns, M. (eds.) SOCASE 2009. LNCS, vol. 5907, pp. 56-70. Springer, Heidelberg (2009) 